\title{
Verzeichnis der Rednerinnen und Redner
}

von Arnim, Hans Herbert 109

Bezemek, Christoph 224

Bickenbach, Christian 112, 465

Classen, Claus Dieter 211

Diggelmann, Oliver 212

Dietz, Andreas 114

Dörr, Dieter 108

Durner, Wolfgang 216

Eberhard, Harald 114

Engel, Christoph 214, 466

Fechner, Frank 106

Fehling, Michael 227

Franzius, Claudio 213, 466

Funke, Andreas 475

Gärditz, Klaus Ferdinand 223

Germann, Michael 218

Griller, Stefan 346

Gröschner, Rolf 225

Groß, Thomas 467

Hain, Karl-E. 106

Hofmann, Rainer 111

Hwang, Shu-Perng 343

Isensee, Josef 113

Jouanjan, Olivier 482

Kempny, Simon 343

Kirchhof, Paul 339

Kirste, Stephan 228

Kischel, Uwe 344

Klein, Tonio 110, 331, 470

Kley, Andreas 230
Knauff, Matthias 475

Kneihs, Benjamin 221

Kotzur, Markus 117, 336

Leisner-Egensperger, Anna 110, 341

Lepsius, Oliver 225, 328

Luther, Jörg 221, 342

Magen, Stefan 120

Mahlmann, Matthias 335

Masing, Johannes 473

Mayer, Franz 333

Murswiek, Dietrich 217

Nowrot, Karsten 118, 332

Puttler, Adelheid 105

Reimer, Franz 479

Ress, Georg 337

Sacksofsky, Ute 330

Saurer, Johannes 341

Schoch, Friedrich 222

Schönberger, Christoph 471

Schröder, Ulrich Jan 115, 228

Somek, Alexander 220, 327

Spiecker, Indra 122

Stelkens, Ulrich 476

Tschentscher, Axel 212

Ullrich, Norbert 112

Vöneky, Silja 119

Volkmann, Uwe 116, 219, 469

Wißmann, Hinnerk 215, 478 
